Math. Model. Nat. Phenom.

Vol. 7, No. 1, 2012, pp. 203-234

DOI: $10.1051 / \mathrm{mmnp} / 20127109$

\title{
Stability Analysis of Cell Dynamics in Leukemia
}

\author{
H. Özbay ${ }^{1 *}$, C. Bonnet ${ }^{2}$, H. Benjelloun ${ }^{3}$ and J. Clairambault ${ }^{4,5}$ \\ ${ }^{1}$ Dept. of Electrical and Electronics Eng., Bilkent University, Ankara, 06800, Turkey \\ ${ }^{2}$ INRIA Saclay - Île-de-France, Equipe DISCO, LSS - SUPELEC \\ 3 rue Joliot Curie, 91192 Gif-sur-Yvette, France \\ ${ }^{3}$ Ecole Centrale Paris, Grande Voie des Vignes, Châtenay-Malabry, France \\ ${ }^{4}$ INRIA Paris-Rocquencourt, Domaine de Voluceau, B.P. 105, 78153 Le Chesney \\ ${ }^{5}$ INSERM team U 776 "Biological Rhythms and Cancers", Hôpital Paul-Brousse \\ 14 Av. Paul-Vaillant-Couturier, 94807 Villejuif, France
}

\begin{abstract}
In order to better understand the dynamics of acute leukemia, and in particular to find theoretical conditions for the efficient delivery of drugs in acute myeloblastic leukemia, we investigate stability of a system modeling its cell dynamics. The overall system is a cascade connection of sub-systems consisting of distributed delays and static nonlinear feedbacks. Earlier results on local asymptotic stability are improved by the analysis of the linearized system around the positive equilibrium. For the nonlinear system, we derive stability conditions by using Popov, circle and nonlinear small gain criteria. The results are illustrated with numerical examples and simulations.
\end{abstract}

Key words: acute leukemia, distributed delays, global stability, absolute stability

AMS subject classification: 93D10, 93D15, 93B52, 93B60, 93C80

\section{Introduction}

In this paper we consider a mathematical model of hematopoietic cell dynamics, developed in a series of papers by Adimy et al., [2, 4, 5, 8], based on earlier models of Mackey and his colleagues, $[15,23,30,34,37,38]$. One of the most recent studies in this line of work is [5], where a system with distributed delay is considered with applications to acute myelogenous leukemia (AML).

${ }^{*}$ Corresponding author. E-mail: hitay@bilkent.edu.tr 
This is a nonlinear system consisting of several compartments connected in series. Equilibrium conditions are studied and local stability analysis for different equilibria is done in [5], where sufficient conditions for local asymptotic stability are obtained using earlier results of [8]. Later, in [52] a necessary and sufficient condition is obtained for a particular choice of the distributed delay kernel for the linearized system.

The main practical interest of studying such stability conditions is to investigate possible stabilizing procedures that may offer guidelines for theoretical therapeutic drug infusion schedules in AML. AML is characterized by uncontrolled proliferation together with a blockade of differentiation. In the special case of promyelocytic acute leukemia (AML3 in the FAB classification), it is known and universally used in the clinic that the combination of a cytotoxic drug and of a redifferentiating agent, two drugs with completely different mechanisms, cures $80 \%$ of patients [28], which unfortunately is not the case of other types of AML. Our goal is firstly to give theoretical, as opposed to empirical, conditions under which this known treatment will be efficient, and secondly to supply biomedical collaborators, who perform pharmacological experiments on leukemic cell cultures, with a rationale to design new therapeutic schemes on various forms of AML. Indeed, one may note that apart from promyelocytic leukemia [28], the pharmacological treatments of AML have remained almost at a standstill in the past forty years [60], new targeted therapies being still under investigation and the only sure way to cure AML being bone marrow transplant [33]. In such context, there is reasonable room for theoretical methods to help better understanding of the disease dynamics and possible therapeutic advances.

Various aspects of linear systems with distributed delays have been studied earlier, [26, 43, $45,46,64,65]$. In this paper, by using a frequency domain analysis, we improve the sufficient conditions of $[5,52]$. For this purpose, we study the gain margin for the linear models around the "positive equilibrium." There are many equilibria for this nonlinear system; here, we concentrate on a particular one which gives non-zero cell count in each compartment of the model proposed by [5]. In this sense, we extend our earlier work [52] to cover all possible cases of parameter values in the linearized model.

The second part of our main results deals with stability analysis of the compartmental nonlinear system. For delay systems with uncertain nonlinearity, primary tools of stability analysis have been the nonlinear small gain condition, circle criterion and Popov criterion, [16, 29, 56, 58], see also $[10,11,48,17]$ and their references. Here, we show that the system studied in [5] can be put within the framework of these methods.

In Section 2. the mathematical model of cell dynamics is described. The main results are presented in Section 3. Numerical examples and simulations are given in Section 4. Concluding results are made in Section 5. 


\section{Mathematical Model of Cell Dynamics in Leukemia}

\subsection{Biological Preliminaries}

The formation of blood cells is called hematopoiesis. It is initiated in the bone marrow by the hematopoietic stem cells (HSCs). HSCs can proliferate, self renew and differentiate into multiple lineages. Proliferation is the cell cycle (growth phase). At the end of this cycle, cell division occurs and two types of cells are produced: daughter cells with the same biological properties as the parent (self-renewal), and progenitors; these are cells that are precursors of the three blood lineages: red blood cells, white blood cells and platelets. When they have reached maturity, they are released into the blood. The production of progenitors at cell division is called differentiation. Progenitors can proliferate and differentiate (in some cases they may self renew or become stem cells, see below for more discussion).

Similar to normal hematopoiesis, leukemia (also known as "blood cancer" characterized by uncontrolled proliferation of blood cells) is caused by leukemia stem cells (LSCs), whose progenitors are capable of self renewal, see Figure 1, [32, 41]. There are both acute and chronic versions of the myelogenous and lymphoblastic leukemia (AML, CML, ALL, CLL).

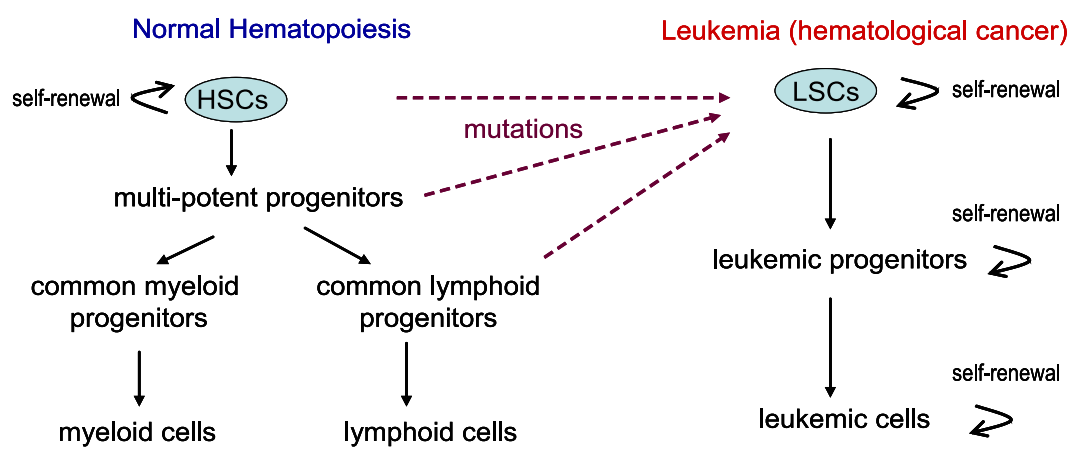

Figure 1: Hematopoiesis and Leukemia.

LSCs are discovered in humans in 1997, [13]. Since then, many other cancer stem cells (CSCs) have been identified: breast cancer in 2003, brain tumor in 2004, and later, colon, prostate, lung and liver CSCs have followed, [12]. Recently, it has been reported that, in some types of cancers, progenitor cells may become stem cells (this is called plasticity), [27], but for the mathematical model of cell dynamics in leukemia our basis will be Figure 1, which is a simplified version of Figure 1 of [41], where further justifying references can be found.

In summary, in normal hematopoiesis and leukemia, there are at least three different populations of cells: (1) HSCs/LSCs, (2) Progenitors, and (3) Myeloid/Lymphoid/Leukemic cells. Cells in population $(i)$ are differentiating to give rise to cells in population $(i+1)$. This is the basis of the "compartmental" dynamical model considered in the paper.

Identification of LSCs is possible by detection of cluster destination (CD) molecules CD34 and CD38 (these are certain types of cell surface proteins). In 1997, [13] have shown that a 
phenotype denoted by CD34+CD38- is the main feature of LSCs. Since then, more detailed studies have been performed and other CD molecules have been determined to be important factors in the identification of LSCs and leukemia progenitors, primarily CD123 (IL-3R $\alpha$ ), CD90 (Thy-1), CD117 (C-Kit), CD135 (Flt-3) and CD33 (Siglec-3), see e.g. [31, 63, 69]. In certain types of AML, one might characterize ([40]) the LSCs, progenitors and leukemia cells with the following differentiation path:

\section{$\mathrm{CD} 34+\mathrm{CD} 38-\mathrm{CD} 33-\rightarrow \mathrm{CD} 34+\mathrm{CD} 38+\mathrm{CD} 33-\rightarrow \mathrm{CD} 34+\mathrm{CD} 38+\mathrm{CD} 33+$}

Hence, one might be able to identify the percentage of populations in three different compartments by detecting these $\mathrm{CD}$ molecules. Of course, it is possible to increase the complexity level and the number of compartments by including CD123, CD90 and other factors. In recent studies, different stages of differentiation are discussed and model parameters are estimated: it is predicted that at least 31 divisions occur between HSC and the circulating blood cells, [20], but for practical purposes 4 to 8 compartment models are sufficient to diagnose CML in humans, [54].

The system we consider here comes from [5], where the mathematical model is based on earlier studies [2]-[9], see also [15, 22, 30, 34, 39, 49, 57]. In the literature, there are many other compartmental dynamical models looking similar to the model of [5]. Many of these do not contain time delays (time spent in the growth phase) or consider point delays only (time spent in the proliferation phase is the same for each cell in a compartment). One of the first mathematical models of hematopoiesis is a single compartment system with a time delay, [37], which is further refined in [38], separating the cell population of the single compartment into two: proliferating cells (those in the growth phase) and quiescent (non-proliferating) cells. See also the earlier paper [67]. Two compartment and four compartment delay-free models of leukemia dynamics can be found in [19] and in [44], respectively. Two coupled delay differential equation model proposed in [38] has been re-considered later by many scientists. Its parameters are estimated in [34] and stability analysis is performed in [1, 68]. This model has been improved in [15] by considering two additional compartments. Similar dynamical models are used for analyzing erythropoiesis (production of red blood cells) [35], and for investigating therapeutic methods in acute lymphoblastic leukemia (ALL), [47]; see also the review [24]. More recently, PDE based models of cell dynamics in hematopoiesis have been studied in $[3,7,36,61]$.

\subsection{Dynamical System Equations}

Consider the compartmental classification of cell populations as discussed in Section 2.1. At each compartment, cell population can be further categorized as proliferating and quiescent (nonproliferating) cells. Let $x_{i}$ denote the quiescent cell population and $y_{i}$ denote the proliferating cell population in compartment $i$. In leukemia, LSCs, progenitors and leukemic cells can self-renew. Therefore, at the cell division in compartment $i$, some portion of daughter cells will be part of $x_{i}$ (this is self-renewal; the newborn cells go to the quiescent phase, and at any given time, certain percentage of the quiescent cells move to the growth phase; this is determined by the "re-introduction function", [38]) and other daughter cells will contribute to $x_{i+1}$ (differentiation). Let $K_{i}$ be the probability of differentiation, $0<K_{i}<1$. So, the rate at which $x_{i}$ is increased, due to the division 
of cells in $y_{i}$, is $2\left(1-K_{i}\right)$ and the rate at which $x_{i+1}$ is increased, due to the division of cells in $y_{i}$, is $2 K_{i}$. Recall that at each cell division two daughter cells are produced, hence the factor 2 multiplying $\left(1-K_{i}\right)$ and $K_{i}$.

Let $\tau_{i}$ be the maximum possible time spent by a cell in proliferation in compartment $i$ before it divides. Some cells divide before $\tau_{i}$. For example, in the erythropoiesis model of [35], time delay ranges from $5 \mathrm{~h}$ (abnormal fast differentiation) to 10 days (late differentiation), and the nominal value is 2 days. In leukemia as well, we expect a non-uniform time delay. This justifies the distributed delay model given below, see also [24] and its references.

Let $\delta_{i}$ denote the death rate for the quiescent cell population. Similarly, let $\gamma_{i}$ denote death rate for the proliferating cell population (certain cells in proliferation die before they have a chance to divide, this is called apoptosis). Then, for each compartment $i \in\{1, \ldots, n\}$ the dynamical system equations are in the form

$$
\begin{aligned}
\dot{x}_{i}(t)=-\delta_{i} x_{i}(t)- & w_{i}(t)+2 L_{i} \int_{0}^{\tau_{i}} e^{-\gamma_{i} a} f_{i}(a) w_{i}(t-a) d a \\
& +2 K_{i-1} \int_{0}^{\tau_{i-1}} e^{-\gamma_{i-1} a} f_{i-1}(a) w_{i-1}(t-a) d a \\
\dot{y}_{i}(t)= & -\gamma_{i} y_{i}(t)+w_{i}(t)-\int_{0}^{\tau_{i}} e^{-\gamma_{i} a} f_{i}(a) w_{i}(t-a) d a
\end{aligned}
$$

where, $L_{i}:=\left(1-K_{i}\right)$, with $K_{0}=0$,

$$
w_{i}(t):=\beta_{i}\left(x_{i}(t)\right) x_{i}(t),
$$

$\beta_{i}(\cdot)$ is the re-introduction function from quiescent subpopulation into the proliferative subpopulation and $f_{i}$ is the mitosis (cell division) probability density. Note that mitosis occurs before the age limit $\tau_{i}$, and

$$
f_{i}(a) \geq 0 \text { for all } a \in\left[0, \tau_{i}\right] \text { and } \int_{0}^{\tau_{i}} f_{i}(a) d a=1 .
$$

The compartmental model is illustrated in Figure 2, and each compartment $\Sigma_{i}$ is depicted in Figure 3, where $u_{i-1}(t)$ for $t \geq 0$, and the initial conditions $x_{i}(a)$ for $a \in\left[-\tau_{i}, 0\right]$, are the inputs and $x_{i}(t), y_{i}(t), u_{i}(t)$, for $t>0$, are the outputs, with $u_{0}=0$. The subsystem represented by the transfer function $G_{i}(s)$ is a linear time-invariant system with distributed delay, whose impulse response is

$$
\begin{gathered}
g_{i}(t):=e^{-\gamma_{i} t} f_{i}(t) \text { for } t \in\left[0, \tau_{i}\right] \text { and } g_{i}(t)=0 \text { for } t>\tau_{i} \text {, i.e., } \\
G_{i}(s)=\int_{0}^{\tau_{i}} e^{-\gamma_{i} t} f_{i}(t) e^{-s t} d t
\end{gathered}
$$

As noted in [5], distributed delay terms in (2.1) come from integration of partial differential equations representing population dynamics. Moreover, the population of proliferating cells $y_{i}$ has no impact on the dynamics of the resting cells. This fact is clearly observed in Figure 3: the internal subsystem $\Sigma_{y i}$ generating $y_{i}$ is a first order linear time invariant system which has no impact on the remaining internal signals of $\Sigma_{i}$. In fact $\Sigma_{y i}$ can be thought as a separate system with input 


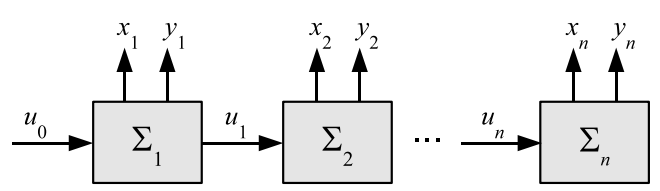

Figure 2: Compartmental Representation of the System.

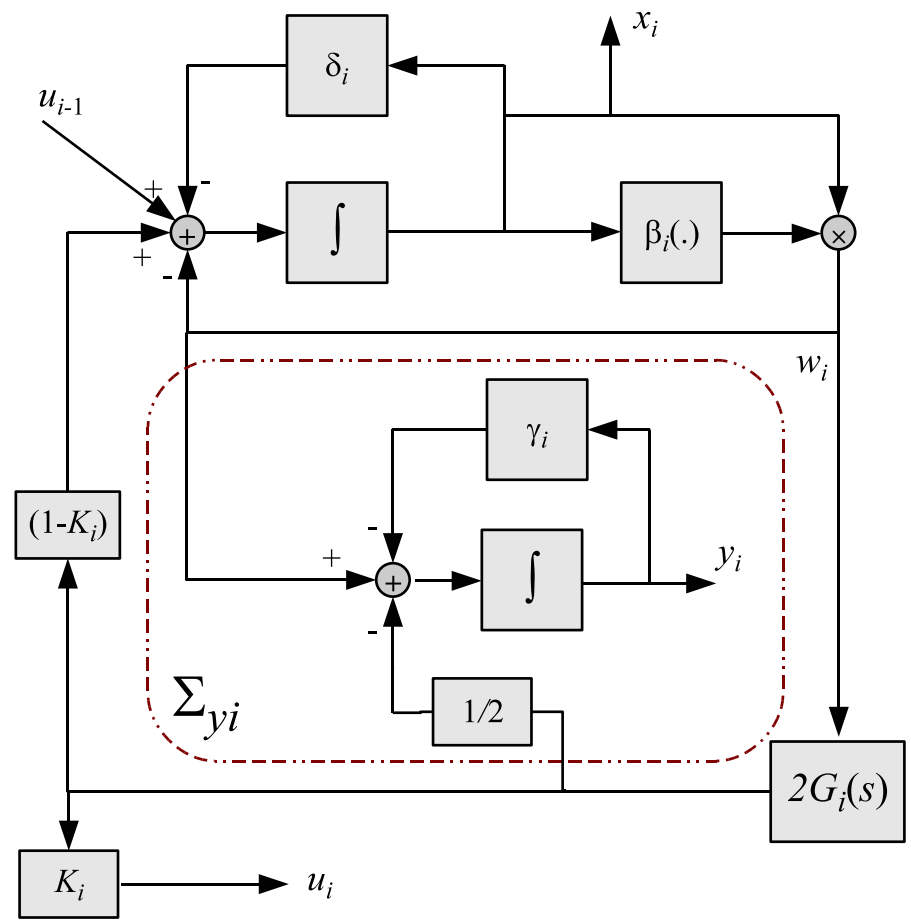

$i$ : compartment number

$x_{i}$ : quiescent cells

$y_{i}:$ proliferating cells

$u_{i}:$ differentiated cells

$w_{i}$ : cells starting to proliferate

$\delta_{i}$ : death rate at the resting phase

$\gamma_{i}$ : death rate at the growth phase

$\beta_{i: \text { re-introduction function }}$

$K_{i}:$ probability of differentiation

$G_{i}$ : dynamics of the growth phase

$\Sigma_{y i}:$ system representing the population dynamics of the proliferating cells

$\int$ : integration operator

$\otimes:$ multiplication operation

† : addition operation

Figure 3: Internal Structure of the Compartment $\Sigma_{i}$.

$\left(I-G_{i}\right) w_{i}$. Then, as long as $\gamma_{i}>0$, the signal $y_{i}$ tracks $\left(I-G_{i}\right) w_{i}$. Therefore, we concentrate on stability analysis of the system represented by equations (2.1) and (2.3) only.

We make the following assumption, which implies that (2.1)-(2.3) admit unique non-negative solution $\left\{x_{i}(t), y_{i}(t), w_{i}(t)\right\}$, for $i=1, \ldots, n$; see e.g. [5, 8] and their references.

Assumption 1. The function $\beta_{i}(\cdot)$ is a differentiable and uniformly decreasing function with $\beta_{i}(0)>0$ and $\beta_{i}(x) \rightarrow 0$ as $x \rightarrow \infty$. The function $f_{i}(\cdot)$ is such that $G_{i}$ is strictly proper, i.e., $G_{i}(s) \rightarrow 0$ as $|s| \rightarrow \infty$. The initial condition $x_{i}(\theta)$ is a continuous function of $\theta \in\left[\tau_{i}, 0\right]$.

Typical selection of $\beta_{i}$ is in the form

$$
\beta_{i}(x)=\frac{\beta_{i}(0)}{1+b_{i} x^{N_{i}}}
$$

where $\beta_{i}(0)>0, b_{i}>0$ and $N_{i}$ is an integer greater or equal to 2 (see [38], and also [15] and [24] for biological justifications and further details). 
In this paper we are interested in a form of $f_{i}$ considered in [52]:

$$
f_{i}(a)=\frac{m_{i}}{e^{m_{i} \tau_{i}}-1} e^{m_{i} a}, \quad a \in\left[0, \tau_{i}\right] \quad m_{i}>\gamma_{i}
$$

which leads to

$$
G_{i}(s)=q_{i} \frac{1-e^{-\tau_{i}\left(s-r_{i}\right)}}{\left(s-r_{i}\right)}
$$

where $q_{i}=m_{i} /\left(e^{m_{i} \tau_{i}}-1\right)>0$ and $r_{i}=m_{i}-\gamma_{i}>0$. Note that $G_{i} \in \mathcal{H}_{\infty}$ and it does not have a pole, in fact $G_{i}\left(r_{i}\right)=q_{i} \tau_{i}$. Some of the results in the next section will be given for generic $G_{i}(s)$ satisfying Assumption 1, and some specific results will be given for the choice (2.7). We should also note that $G_{i},(2.7)$, is a stable system whose impulse response is non-negative, therefore,

$$
\left\|G_{i}\right\|_{\infty}=\sup _{\omega}\left|G_{i}(j \omega)\right| \leq \sup _{\omega} \int_{0}^{\infty}\left|g_{i}(t)\right|\left|e^{-j \omega t}\right| d t=\int_{0}^{\infty} g_{i}(t) d t=G_{i}(0) \leq\left\|G_{i}\right\|_{\infty} .
$$

Thus $G_{i}(0)=\left\|G_{i}\right\|_{\infty}$.

As mentioned in [52], the choice (2.6) corresponds to a truncated exponential term for the mitosis function. In this model of mitosis, cells are authorized to divide with a probability that increases exponentially with age in the division cycle (most of the division occurs just before the age limit $\tau_{i}$ ). Whereas in [5] this division probability is taken as a simple Dirac mass at the end of the cycle, i.e. $f_{i}(t)=\delta\left(t-\tau_{i}\right)$, we have chosen in the present study to mimic this biological behavior in a more continuous way, allowing thus cells to divide increasingly with age. Even though this increase is very steep, it seems to us biologically more relevant than a Dirac mass.

Remark 1. When $f_{o}(t):=\delta\left(t-\tau_{i}\right)$ we have $G_{o}(s)=e^{-\tau_{i}\left(s+\gamma_{i}\right)}$ and hence $G_{o}(0)=e^{-\gamma_{i} \tau_{i}}$. On the other hand, when $G_{i}(s)$ is in the form (2.7) we have

$$
G_{i}(0)=\frac{m_{i}}{m_{i}-\gamma_{i}} \frac{e^{\left(m_{i}-\gamma_{i}\right) \tau_{i}}-1}{e^{m_{i} \tau_{i}}-1}
$$

We claim that $G_{i}(0)>G_{o}(0)$ for all $m_{i}>\gamma_{i}$ and $\tau_{i}>0$. It is easy to check that the claim is true if and only if

$$
\frac{e^{m_{i} \tau_{i}}-e^{\tau_{i} \gamma_{i}}}{e^{m_{i} \tau_{i}}-1}>\frac{m_{i}-\gamma_{i}}{m_{i}}
$$

which is equivalent to

$$
\frac{\gamma_{i}}{e^{\gamma_{i} \tau_{i}}-1}>\frac{m_{i}}{e^{m_{i} \tau_{i}}-1}
$$

The condition (2.8) holds for all $m_{i}>\gamma_{i}$ and $\tau_{i}>0$ because the function $x\left(e^{\tau x}-1\right)^{-1}$ is a decreasing function of $x$ when $x>0$ and $\tau>0$. Thus,

$$
G_{i}(0)>e^{-\gamma_{i} \tau_{i}} \text { for all } m_{i}>\gamma_{i} \text { and } \tau_{i}>0
$$

as claimed. The inequality (2.9) will allow us to compare stability results obtained by taking $f_{i}=\delta\left(t-\tau_{i}\right)$ with the results obtained by the choice (2.6). Also, note that when $m_{i} \rightarrow \infty$ the distribution (2.6) approaches to a Dirac mass at $\tau_{i}$, and in this case $G_{i}(0) \rightarrow e^{-\gamma_{i} \tau_{i}}$. 
Clearly, the origin is an equilibrium of the nonlinear system, defined by (2.1)-(2.3), that corresponds to death of all cells. Stability conditions for the case where the origin is the unique equilibrium are obtained in [6]. In this paper we derive stability conditions for a "positive equilibrium point," $\left[\bar{x}_{1}, \ldots, \bar{x}_{n}\right]^{T}$, where all $\bar{x}_{i}$ are strictly positive. There are other equilibrium points where some of the $\bar{x}_{i}$ are zero and some are positive; for details see [5]. We now define

$$
\alpha_{i}:=2 L_{i} \int_{0}^{\tau_{i}} g_{i}(t) d t-1=2 L_{i} G_{i}(0)-1
$$

and make the following additional assumptions.

Assumption 2. We have $\alpha_{i}>0$ for all $i=1, \ldots, n$.

Remark 2. To justify this assumption, let us consider the minimal case for the source term $\beta$ of the proliferating compartment dynamics when only self-renewal is active with no actual input from a non proliferating buffer compartment, and no loss term by differentiation. These are the minimal conditions for actual proliferation, i.e. growth of the proliferative compartment. Then this minimal dynamics is represented by the classical transport equation of population dynamics, structured in age a (see, e.g., [55]; indeed, this equation dates back to McKendrick, see [42]):

$$
\left\{\begin{aligned}
\frac{\partial n}{\partial a}(t, a)+\frac{\partial n}{\partial t}(t, a)+2 L k(a) n(t, a)+\gamma n(t, a) & =0 \\
n(t=0, a) & =n^{0}(a) \\
n(t, a=0) & =2 L \int_{0}^{+\infty} k(y) n(t, y) d y
\end{aligned}\right.
$$

where $k(a)$ is the rate of self-renewal. It is then classical (by integration along characteristics) in population dynamics that the probability density function of mitosis is given by

$$
f(a)=k(a) e^{\int_{0}^{a} k(y) d y},
$$

and (by injection of the solution into the boundary condition) that the leading growth exponent $\lambda$ is the only positive real solution to Lotka's equation

$$
1=2 L \int_{0}^{+\infty} e^{-\lambda a-\gamma a} f(a) d a
$$

Saying that $\alpha_{i}>0$ is then exactly saying that $\lambda>0$, i.e., that the process is a growth process.

Assumption 3. For $i=1$, we have $\beta_{1}(0)>\delta_{1} / \alpha_{1}$.

Under Assumptions 1-3, a unique positive equilibrium exists, see e.g. [5]. It can be computed from the following equations: $\bar{x}_{1}$ is such that

$$
\beta_{1}\left(\bar{x}_{1}\right)=\delta_{1} / \alpha_{1}
$$

and for $i \geq 2$, the equilibrium points $\bar{x}_{i}$ are the unique solutions of

$$
\beta_{i}\left(\bar{x}_{i}\right)=\frac{1}{\alpha_{i}}\left(\delta_{i}-\frac{1}{\bar{x}_{i}} \frac{\bar{x}_{i-1} K_{i-1}\left(1+\alpha_{i-1}\right)}{L_{i-1}} \beta_{i-1}\left(\bar{x}_{i}\right)\right) .
$$


When dealing with local asymptotic stability we consider linearized version of (2.1) around $\bar{x}_{i}$. In particular, we define

$$
\mu_{i}:=\left.\frac{\mathrm{d}}{\mathrm{d} x} x \beta_{i}(x)\right|_{\bar{x}_{i}} .
$$

In $\Sigma_{i}$, if we use the approximation $w_{i} \approx \mu_{i} x_{i}$ then the feedback system obtained has the following characteristic equation

$$
s+\delta_{i}+\mu_{i}-2 L_{i} \mu_{i} G_{i}(s)=0 .
$$

Since $G_{i}(s)$ is assumed to be strictly proper, the linearized system is stable if and only if all the roots of (2.14) are in $\mathbb{C}_{-}$. As noted in [8], $\mu_{i}$ can take any values in $\mathbb{R}$. Clearly, when $\mu_{i}=0$ the system is locally asymptotically stable around the equilibrium $\bar{x}_{i}$, because $\delta_{i}>0$. Therefore, the most interesting case is $\mu_{i} \neq 0$. Because the roots of (2.14) depend on $\mu_{i}$, local stability conditions of Section 3.1. are obtained in terms of this key parameter.

\subsection{Earlier results and summary of contributions}

In the remaining parts of the paper we drop the subscript $i$ for notational convenience, whenever it is clear from the context that we are dealing with the $i$ th subsystem $\Sigma_{i}$. We will consider different stability notions. First, local asymptotic stability means that $x(t) \rightarrow \bar{x}$ as $t \rightarrow \infty$ provided that the initial condition is in a neighborhood of $\bar{x}$, i.e. $\sup _{-\tau \leq \theta \leq 0}|\bar{x}-x(\theta)| \leq \varepsilon_{o}$ for some $\varepsilon_{o}>0$. This result does not say anything about how large the region of attraction, $\varepsilon_{o}$, is. In Section 3.2.3. there is a well defined region around $\bar{x}$ where a sector condition, either (3.34) or (3.34), is satisfied. Absolute stability will imply that as long as the initial condition is within the region where sector condition is satisfied, convergence to the equilibrium is guaranteed. In Sections 3.2.1. and 3.2.2., we will give conditions for global stability, where convergence to the equilibrium is guaranteed with no restriction on the initial condition. Clearly, global stability implies absolute stability which implies local stability.

Earlier results on stability of the system defined in Section 2.2. can be summarized as follows.

(a) In [5] it has been shown that the origin is locally asymptotically stable if $\beta_{i}(0)<\delta_{i} / \alpha_{i}$, for all $i$, and it is unstable if $\beta_{i}(0)>\delta_{i} / \alpha_{i}$ for some $i$.

(b) In $[5,8]$ it is claimed that $\bar{x}>0$ is locally asymptotically stable if

$$
(2 L G(0)+1) \mu+\delta>0 .
$$

While this statement is correct for $-\delta<\mu<0$, it becomes false under Assumptions 2 and 3 , when $\mu>0$ (see (c) below). For $\mu<-\delta$, (2.15) is only a necessary condition for local asymptotic stability (see Section 3.1.2. for necessary and sufficient conditions, (3.9) and (3.10)). Moreover, the condition (2.15) can be very conservative for $-\delta<\mu<0$. In the present paper we illustrate the level of conservatism for the particular choice of $G$ in the form (2.7). 
(c) In [52], for $\mu>0$, it is shown that all the roots of (2.14) are in $\mathbb{C}_{-}$if and only if $\mu<\delta / \alpha$. Note that for $\mu>0$ all positive values of $\delta$ satisfy (2.15), whereas the necessary and sufficient condition of [52] impose a restriction on $\delta$. In the present paper, methods similar to those of [52] are used for the case $\mu<0$.

(d) Most recently, in [6] it is shown that (Theorem 2.1 of [6]) if the origin is the only equilibrium and $\left(2 G_{i}(0)-1\right) \beta_{i}(0)<\delta_{i}$, for all $i$, then the origin is globally asymptotically stable.

(e) In [57], for the point delay version of the above model, conditions for global asymptotic stability of the origin (Theorem 6.4 of [57]) and instability of the positive equilibrium (Theorem 7.2 of [57]) are obtained in terms of the delay values.

The contributions of this paper are as follows:

(i) Stability conditions are obtained for the linearized system around the positive equilibrium with $\mu<0$, using the Nyquist criterion.

(ii) A "small-gain" argument is applied to derive a global asymptotic stability condition for the positive equilibrium.

(iii) Less restrictive conditions of stability, than (ii), are derived for a finite neighborhood of the positive equilibrium. These are obtained from Popov and circle criteria.

\section{Main Results}

In this section we derive new stability conditions for the linearized system and for the nonlinear system under Assumptions 1-3.

\subsection{Stability of the linearized system}

First we consider the linearized version of $\Sigma_{i}$ around $\bar{x}_{i}>0$ and improve the earlier results mentioned above, particularly for the case of $\mu<0$. But we begin with an observation on the case $\mu>0$.

\subsubsection{A remark on the case $\mu>0$}

Recall that in this case the stability condition is

$$
\mu<\delta / \alpha
$$

Proposition 1. The condition (3.1) holds true for all $\beta$ in the form (2.5). 
Proof. Note that at the equilibrium point $\bar{x}$ (for all the compartments, $i=1,2, \ldots, n$ ), by (2.11) and (2.12), we have $\beta(\bar{x}) \leq \delta / \alpha$. Also note that for $\beta$ in the form (2.5) we have

$$
\mu=\left.\frac{\partial}{\partial x} x \beta(x)\right|_{\bar{x}}=\beta(\bar{x})\left(1-N+N \frac{\beta(\bar{x})}{\beta(0)}\right) .
$$

Since $N \geq 2$ and $\beta(\bar{x})<\beta(0)$ we have that

$$
\left(1-N+N \frac{\beta(\bar{x})}{\beta(0)}\right)<1 .
$$

This inequality with $\beta(\bar{x}) \leq \delta / \alpha$ implies that $\mu<(\delta / \alpha)$. Thus, when $\mu>0$, the system is locally asymptotically stable whenever $\beta$ is in the form (2.5).

Note that the selection of cell division probability density $f$ plays an important role in (3.1), since it determines $G(0)$, which affects $\alpha=2 L G(0)-1$. As $\alpha$ increases the allowable $\frac{\delta}{\mu}$ (for local stability) decreases. By the inequality (2.9) derived in Remark 1, we see that taking $f$ in the form (2.6) puts more restriction on the ratio of allowable $(\delta / \mu)$ than taking $f(t)=\delta\left(t-\tau_{i}\right)$.

\subsubsection{Improved stability conditions for $\mu<0$}

Consider the system whose characteristic equation is in the form (2.14) with $\mu<0$. Assume that $G(-(\delta-|\mu|)) \neq 0$ (which is satisfied for $G(s)$ in the form (2.7) when $\delta>|\mu|)$, then (2.14) can be re-written as

$$
1+|\mu| \frac{2 L G(s)}{s+(\delta-|\mu|)}=0
$$

Alternatively, if we assume $2 L G(-\delta) \neq 1$ (which is generically satisfied), then (2.14) can be put in the form

$$
1+|\mu| H(s)=0
$$

where

$$
H(s):=\frac{(2 L G(s)-1)}{(s+\delta)} .
$$

In this section we will use (3.2) for local stability analysis. The equation (3.3) will be used in the next section when the circle and Popov criterion are applied to the nonlinear system.

Local stability conditions can be obtained by using the Nyquist criterion, (see, e.g.,[21, 51]), on the system represented by (3.2) in two different cases: $\underline{\text { Case 1 }}: \delta>|\mu|$; and $\underline{\text { Case 2: }} \delta<|\mu|$.

In Case 1, (3.2) is the characteristic equation of a feedback system formed by two stable systems $2 L G(s)$ and $|\mu| /(s+(\delta-|\mu|))$. Therefore, for local stability, the graph of $\frac{2 L G(j \omega)|\mu|}{j \omega+(\delta-|\mu|)}$, as $\omega$ varies from 0 to $\infty$, should not encircle -1 in the complex plane. Consider $G$ in the form (2.7):

$$
G(s)=q \frac{1-e^{-\tau(s-r)}}{(s-r)}=G(0) \frac{e^{\widehat{\tau}} e^{-\widehat{\tau} \widehat{s}}-1}{\left(e^{\widehat{\tau}}-1\right)(1-\widehat{s})}
$$


where $\widehat{\tau}=r \tau$ and $\widehat{s}=s / r$. In this case, the characteristic equation is

$$
1+\frac{2 L|\mu|}{\delta-|\mu|} G(0) \frac{e^{\widehat{\tau}} e^{-\widehat{\tau} \widehat{s}}-1}{\left(e^{\widehat{\tau}}-1\right)(1-\widehat{s})(1+\eta \widehat{\tau} \widehat{s})}=0
$$

where $\eta:=\tau^{-1}(\delta-|\mu|)^{-1}$. Let us define

$$
\widetilde{G}(s):=(1+\eta \tau s)^{-1} G(0)^{-1} G(s)=\frac{e^{\widehat{\tau}} e^{-\widehat{\tau} \widehat{s}}-1}{\left(e^{\widehat{\tau}}-1\right)(1-\widehat{s})(1+\eta \widehat{\tau} \widehat{s})} .
$$

By analyzing the graph of $\widetilde{G}(j \omega)$, for $\omega \in \mathbb{R}$, we have local stability if and only if

$$
2 L G(0)<\frac{\delta-|\mu|}{|\mu|} k_{\max }
$$

where $k_{\max }:=\left|\widetilde{G}\left(j \omega_{1}\right)\right|^{-1}$ with $\omega_{1}$ being the smallest $\omega>0$ such that $\angle \widetilde{G}(j \omega)=-\pi$, where $\angle$ denotes the phase (angle) function.

Table 1: Values of $k_{\max }$ for different $\widehat{\tau}$ (first row) and $\eta$ (first column).

\begin{tabular}{||l||l|l|l|l|l|l||}
\hline \hline$\eta \backslash \widehat{\tau}$ & $1 / 10$ & 1 & 2 & 4 & 10 & 20 \\
\hline \hline $1 / 4$ & 4.27 & 2.73 & 2.06 & 1.56 & 1.26 & 1.21 \\
\hline 1 & 6.91 & 5.03 & 3.97 & 3.06 & 2.49 & 2.36 \\
\hline 2 & 11.50 & 8.52 & 6.77 & 5.24 & 4.23 & 3.99 \\
\hline 5 & 25.67 & 19.14 & 15.28 & 11.81 & 9.51 & 8.96 \\
\hline \hline
\end{tabular}

The numerical values of $k_{\max }$ for different $\widehat{\tau}=r \tau$ and $\eta$ are shown in Table 1 . Note that for all values of $\eta$ and $\widehat{\tau}$ we have $k_{\max }>1$. On the other hand, when $\delta>|\mu|$, the sufficient condition obtained earlier, (2.15), is equivalent to

$$
2 L G(0)<\frac{\delta-|\mu|}{|\mu|}
$$

Since $k_{\max }>1$, the condition (3.7) is less restrictive than (3.8). Moreover, the level of conservatism in (2.15) can be determined from the values of $k_{\max }$ given in Table 1.

In Case 2, i.e. when $|\mu|>\delta$, the characteristic equation (3.2) becomes

$$
1+\frac{2 L|\mu|}{|\mu|-\delta} G(0) \frac{e^{\widehat{\tau}} e^{-\widehat{\tau} \widehat{s}}-1}{\left(e^{\widehat{\tau}}-1\right)(1-\widehat{s})(\eta \widehat{\tau} \widehat{s}-1)}=0 .
$$

with $\eta:=\tau^{-1}(|\mu|-\delta)^{-1}$. Note that the open loop system is unstable with one pole in the right half plane. For stability of this system the Nyquist graph of $\widehat{k} \widehat{G}(j \omega)$ should encircle -1 once in the counter clockwise direction, where

$$
\widehat{k}:=\frac{2 L|\mu|}{|\mu|-\delta} G(0), \quad \widehat{G}(s):=\frac{e^{\widehat{\tau}} e^{-\widehat{\tau} \widehat{s}}-1}{\left(e^{\widehat{\tau}}-1\right)(1-\widehat{s})(\eta \widehat{\tau} \widehat{s}-1)} .
$$


This is satisfied if and only if the following three conditions are met

$$
\widehat{k}>1 ; \quad\left|\widehat{G}\left(j \omega_{p}\right)\right|<1 / \widehat{k} ;\left.\quad \frac{\mathrm{d}}{\mathrm{d} \omega} \angle \widehat{G}(j \omega)\right|_{\omega=0}>0
$$

where $\omega_{p}$ is the smallest $\omega>0$ such that $\angle \widehat{G}(j \omega)=-\pi$. The first condition means that $\widehat{k} \widehat{G}(0)<$ -1 . The third condition means that $\widehat{k} \widehat{G}(j \omega)$ moves towards the 3rd quadrant of the complex plane as $\omega$ increases from zero to small positive values. The second condition implies that when the imaginary part of $\widehat{k} \widehat{G}(j \omega)$ becomes zero for the first time (as $\omega$ increases from 0 ) its magnitude is less than 1 . Thus the above three conditions is equivalent to having the graph of $\widehat{k} \widehat{G}(j \omega)$ encircle -1 once in the counterclockwise direction as $\omega$ increases from $-\infty$ to $+\infty$.

The third condition is equivalent to

$$
\eta>\left(1-e^{-\widehat{\tau}}\right)^{-1}-\widehat{\tau}^{-1}
$$

and the first two conditions can be combined as

$$
\frac{|\mu|-\delta}{|\mu|}<2 L G(0)<\frac{|\mu|-\delta}{|\mu|} k_{\max , 2},
$$

where $k_{\max , 2}=1 /\left|\widehat{G}\left(j \omega_{p}\right)\right|$ can be computed numerically using Matlab or Scilab. The numerical values of $k_{\max , 2}$ for different $\widehat{\tau}$ and $\eta$ are shown in Table 2 (here $\star$ indicates that for these values of $\widehat{\tau}$ and $\eta$ the condition (3.9) is not satisfied).

Table 2: Values of $k_{\max , 2}$ for different $\widehat{\tau}$ (first row) and $\eta$ (first column).

\begin{tabular}{||l||l|l|l|l|l|l||}
\hline \hline$\eta \backslash \widehat{\tau}$ & $1 / 10$ & $4 / 10$ & 1 & 2 & 5 & 10 \\
\hline \hline 0.8 & 2.21 & 2.01 & 1.70 & 1.38 & $\star$ & $\star$ \\
\hline 0.9 & 2.66 & 2.41 & 2.04 & 1.65 & 1.18 & $\star$ \\
\hline 1.0 & 3.10 & 2.81 & 2.37 & 1.92 & 1.38 & 1.17 \\
\hline \hline
\end{tabular}

The analysis technique used in this section, namely Nyquist stability condition, gives exact non-conservative results of the stability of the linearized system considered here. The same result could have been obtained from other techniques used in the literature for delay systems, see for example $[14,59]$ and their references.

\subsection{Stability analysis for the nonlinear system}

In this section we discuss stability of the $i$ th compartment $\Sigma_{i}$, whose structure is shown in Figure 3 . Under Assumptions 1-3, we have a positive equilibrium $\bar{x}_{i}>0$. Let us define

$$
\widetilde{x}_{i}(t):=x_{i}(t)-\bar{x}_{i} \quad \text { and } \quad \widetilde{w}_{i}(t):=w_{i}(t)-\bar{w}_{i}
$$


where $\bar{w}_{i}:=\beta_{i}\left(\bar{x}_{i}\right) \bar{x}_{i}$. Under equilibrium conditions (2.11) and (2.12), the system (2.1) can be transformed to

$$
\frac{d}{d t} \widetilde{x}_{i}(t)=-\delta_{i} \widetilde{x}_{i}(t)-\widetilde{w}_{i}(t)+2 L_{i} \int_{0}^{\tau_{i}} e^{-\gamma_{i} a} f_{i}(a) \widetilde{w}_{i}(t-a) d a+\widetilde{u}_{i-1}(t)
$$

where

$$
\widetilde{u}_{i-1}(t):=2 K_{i-1} \int_{0}^{\tau_{i-1}} e^{-\gamma_{i-1} a} f_{i-1}(a) \widetilde{w}_{i-1}(t-a) d a .
$$

Using the new coordinates (3.11), the origin (i.e. $\widetilde{x}_{i}=0$ ) is the equilibrium of the system (3.12), whose feedback diagram is shown in Figure 4, where $\psi_{i}$ is the static nonlinearity defined by $\psi_{i}\left(\widetilde{x}_{i}\right)=\widetilde{w}_{i}$, more precisely

$$
\psi_{i}\left(\widetilde{x}_{i}\right)=\left(\widetilde{x}_{i}+\bar{x}_{i}\right) \beta_{i}\left(\widetilde{x}_{i}+\bar{x}_{i}\right)-\bar{x}_{i} \beta_{i}\left(\bar{x}_{i}\right) .
$$

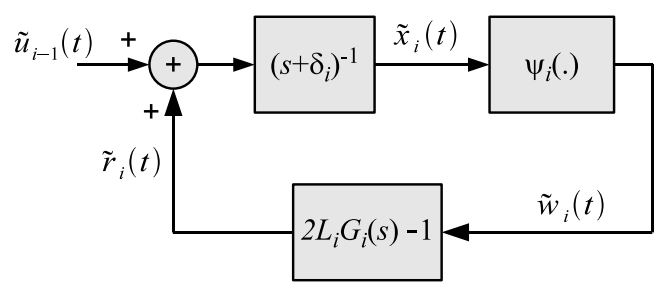

Figure 4: Feedback system $\widetilde{\Sigma}_{i}$ with equilibrium at the origin.

Let us now define the gain of the nonlinear block as

$$
\rho_{i}:=\inf \left\{R \in \mathbb{R}:\left|\psi_{i}\left(\widetilde{x}_{i}\right)\right| \leq R\left|\widetilde{x}_{i}\right| \quad \forall \widetilde{x}_{i} \in\left(-\bar{x}_{i}, \infty\right)\right\} .
$$

Recall from the definition (2.13) that $\mu_{i}$ is the slope of the nonlinear block $\psi_{i}\left(\widetilde{x}_{i}\right)$ at $\widetilde{x}_{i}=0$; whereas $\rho_{i}$ is the least possible slope of all the lines which pass through the origin and stay above $\psi_{i}\left(\widetilde{x}_{i}\right)$ for all $\widetilde{x}_{i}>0$ and under $\psi_{i}\left(\widetilde{x}_{i}\right)$ for all $\widetilde{x}_{i}<0$. So, we have $\rho_{i} \geq\left|\mu_{i}\right|$ for both cases where $\mu_{i}>0$ and $\mu_{i}<0$.

Considering the linear part of the system shown in Figure 4, we can write the solution $\widetilde{x}_{i}(t)$ in terms of the initial conditions and the external input as

$$
\widetilde{x}_{i}(t)=e^{-\delta_{i} t} \widetilde{x}_{i}(0)+\int_{-\tau_{i}}^{t} e^{-\delta_{i}(t-a)} \widetilde{r}_{i}(a) d a+\int_{-\tau_{i-1}}^{t} e^{-\delta_{i}(t-a)} \widetilde{u}_{i-1}(a) d a
$$

where

$$
\widetilde{r}_{i}(a)=2 L_{i} \int_{0}^{\tau_{i}} g_{i}(\theta) \widetilde{w}_{i}(a-\theta) d \theta-\widetilde{w}_{i}(a), \text { for } a>-\tau_{i}
$$

and it is determined starting from the initial condition

$$
\widetilde{w}_{i}(\theta)=\left(\widetilde{x}_{i}(\theta)+\bar{x}_{i}\right) \beta_{i}\left(\widetilde{x}_{i}(\theta)+\bar{x}_{i}\right)-\bar{x}_{i} \beta_{i}\left(\bar{x}_{i}\right) \text { for } \theta \in\left[-\tau_{i}, 0\right] .
$$




\subsubsection{Analysis for the first compartment}

For $\widetilde{\Sigma}_{1}$ shown in Figure 4 we have $\widetilde{u}_{0} \equiv 0$. Note that, in (3.17), $\widetilde{r}_{i}$ is obtained by passing $\widetilde{w}_{i}$ from the filter $(2 L G(s)-1)$. So, (3.16) can be re-written as (dropping the subscript for notational convenience)

$$
\widetilde{x}(t)=e^{-\delta t} \widetilde{x}(0)+\int_{-\tau}^{t} h(t-a) \widetilde{w}(a) d a
$$

where

$$
h(t)=\left(2 L \int_{0}^{\min \{t, \tau\}} g(a) e^{\delta a} d a-1\right) e^{-\delta t} \quad \text { for } t \geq 0
$$

is the impulse response of the transfer function $H(s)=(s+\delta)^{-1}(2 L G(s)-1)$.

Using (3.15) we can find an upper bound for the magnitude of $\widetilde{x}(t)$ in (3.19) as

$$
|\widetilde{x}(t)| \leq e^{-\delta t}|\widetilde{x}(0)|+\rho \int_{-\tau}^{t}|h(t-a)||\widetilde{x}(a)| d a .
$$

Recall Assumption 1 (the part on the initial condition) and define

$$
\begin{gathered}
\chi_{k}:=\max _{(k-1) \tau \leq t \leq k \tau}|\widetilde{x}(t)| \\
h_{k}(\tau, \delta, L, g):=\int_{k \tau}^{(k+1) \tau}|h(t)| d t .
\end{gathered}
$$

By definition, we see that

$$
\begin{gathered}
h_{0}=\int_{0}^{\tau}\left|2 L \int_{0}^{t} g(a) e^{\delta a} d a-1\right| e^{-\delta t} d t \\
h_{k}=\widetilde{\alpha} \frac{\left(1-e^{-\delta \tau}\right)}{\delta} e^{-k \delta \tau} \quad k \geq 1,
\end{gathered}
$$

where

$$
\widetilde{\alpha}:=\left(2 L \int_{0}^{\tau} g(a) e^{\delta a} d a-1\right)
$$

From (3.21) we have

$$
\chi_{k} \leq e^{-\delta \tau(k-1)}|\widetilde{x}(0)|+\rho \sum_{i=0}^{k} h_{i} \chi_{k-i} .
$$

If

$$
\rho h_{0}<1
$$

then (3.26) leads to

$$
\chi_{k} \leq \frac{1}{1-\rho h_{o}}\left(e^{-\delta \tau(k-1)}|\widetilde{x}(0)|+\rho \sum_{i=1}^{k} h_{i} \chi_{k-i}\right)
$$


Define a new variable $\xi_{k}$ satisfying

$$
\xi_{k}=\frac{1}{1-\rho h_{o}}\left(e^{-\delta \tau(k-1)}|\widetilde{x}(0)|+\rho \sum_{i=1}^{k} h_{i} \xi_{k-i}\right) \quad \text { for } \quad k \geq 1
$$

with the initial condition $\xi_{0}=\chi_{0}$. Clearly, we have $\chi_{k} \leq \xi_{k}$ for all $k \geq 1$. Note that (3.29) is a discrete time linear system (see e.g. [50]) whose unit pulse response is

$$
\left(1-\rho h_{0}\right)^{-1} \rho \widetilde{\alpha} \frac{\left(1-e^{-\delta \tau}\right)}{\delta} e^{-\delta \tau k}, \text { for } k \geq 1,
$$

and excited by the input

$$
\left(1-\rho h_{0}\right)^{-1} e^{\delta \tau}|\widetilde{x}(0)| e^{-\delta \tau k}, \text { for } k \geq 1
$$

Therefore, we conclude that

$$
\max _{(k-1) \tau \leq t \leq k \tau}|\widetilde{x}(t)|=\chi_{k} \leq c_{o} e^{-\delta \tau k}+c_{1} \tau k e^{-\delta \tau k} \quad \text { for all } \quad k \geq 1
$$

for some constants $c_{o}, c_{1} \in \mathbb{R}$, see [50].

Definition 2. We say that a bounded signal $\widetilde{x}(t)$ converges to zero exponentially at a rate $\lambda>0$ if there exist $K>0$ and an integer $m \geq 0$, such that $|\widetilde{x}(t)| \leq K\left(1+t^{m}\right) e^{-\lambda t}$ for all $t \geq 0$.

The above discussion can now be summarized as follows.

Proposition 3. Consider the system $\widetilde{\Sigma}_{1}$ shown in Figure 4, with $\widetilde{u}_{0}=0$. Suppose that $\rho_{1}$ defined in (3.15) is finite and let

$$
h_{0}\left(\tau_{1}, \delta_{1}, L_{1}, g_{1}\right):=\int_{0}^{\tau_{1}}\left|2 L_{1} \int_{0}^{t} g_{1}(a) e^{\delta_{1} a} d a-1\right| e^{-\delta_{1} t} d t
$$

If

$$
h_{0}\left(\tau_{1}, \delta_{1}, L_{1}, g_{1}\right)<1 / \rho_{1}
$$

then $\widetilde{x}_{1}(t)$ is bounded and converges to zero exponentially at a rate $\delta_{1}$. Since $\left|\widetilde{w}_{1}(t)\right| \leq \rho_{1}\left|\widetilde{x}_{1}(t)\right|$ and

$$
\widetilde{r}_{1}(t)=2 L_{1} \int_{0}^{\tau_{1}} g_{1}(a) \widetilde{w}_{1}(t-a) d a-\widetilde{w}_{1}(t)
$$

we also have that $\widetilde{w}_{1}(t)$ and $\widetilde{r}_{1}(t)$ remain bounded and converge to zero exponentially at a rate $\delta_{1}$. 


\subsubsection{Analysis for the $k$ th compartment for $k \geq 2$}

For $\widetilde{\Sigma}_{2}$ the input $\widetilde{u}_{1}(t)$ comes from the internal signals $\widetilde{r}_{1}(t)$ and $\widetilde{w}_{1}(t)$ of $\widetilde{\Sigma}_{1}$ as $\widetilde{u}_{1}=K L^{-1}\left(\widetilde{r}_{1}+\right.$ $\left.\widetilde{w}_{1}\right)$. Now using (3.16) we have

$$
\left|\widetilde{x}_{2}(t)\right| \leq e^{-\delta_{2} t}\left|\widetilde{x}_{2}(0)\right|+\rho_{2} \int_{-\tau_{2}}^{t}\left|h_{2}(t-a)\right|\left|\widetilde{x}_{2}(a)\right| d a+\int_{-\tau_{1}}^{t} e^{-\delta_{2}(t-a)}\left|\widetilde{u}_{1}(a)\right| d a
$$

Note that under the condition (3.31) the signal $\widetilde{u}_{1}$ is bounded and converges to zero exponentially at a rate $\delta_{1}$. Therefore

$$
e^{-\delta_{2} t}\left|\widetilde{x}_{2}(0)\right|+\int_{-\tau_{1}}^{t} e^{-\delta_{2}(t-a)}\left|\widetilde{u}_{1}(a)\right| d a
$$

is a bounded signal converging to zero exponentially at a rate $\widetilde{\delta}_{2}:=\min \left\{\delta_{1}, \delta_{2}\right\}$. Now using arguments similar to those (3.22)-(3.30) it is easy to see that if

$$
h_{0}\left(\tau_{2}, \delta_{2}, L_{2}, g_{2}\right)<1 / \rho_{2}
$$

then $\widetilde{x}_{2}(t)$ is a bounded signal converging to zero at a rate $\widetilde{\delta}_{2}$. Repeating this argument for successive compartments until $\widetilde{\Sigma}_{k}$ we obtain the following result.

Proposition 4. Consider the system $\widetilde{\Sigma}_{k}$ shown in Figure 4. Suppose $\rho_{1}, \ldots, \rho_{k}$ defined in (3.15) are finite and let

$$
h_{0}\left(\tau_{i}, \delta_{i}, L_{i}, g_{i}\right):=\int_{0}^{\tau_{i}}\left|2 L_{i} \int_{0}^{t} g_{i}(a) e^{\delta_{i} a} d a-1\right| e^{-\delta_{i} t} d t \quad \text { for } i=1, \ldots, k .
$$

If

$$
h_{0}\left(\tau_{i}, \delta_{i}, L_{i}, g_{i}\right)<1 / \rho_{i} \quad \forall i=1, \ldots, k
$$

then $\widetilde{x}_{k}(t), \widetilde{w}_{k}(t), \widetilde{r}_{k}(t)$ remain bounded for all times and they converge to zero exponentially at a rate $\widetilde{\delta}_{k}:=\min \left\{\delta_{1}, \ldots, \delta_{k}\right\}$.

\subsubsection{Application of the circle and Popov criteria}

The result given above depend on the small gain condition (3.33), where $\rho_{i}$ satisfy (3.15). An alternative result can be found by putting the system of Figure 4 into the framework of the circle (or Popov) criterion. For this purpose, let us consider the first compartment, $\widetilde{\Sigma}_{1}$; recall the definitions (3.11) and assume that (suppressing the subscript) there exists $\widetilde{\rho}>0$ satisfying the sector condition

$$
\text { for } \mu>0: \quad 0<\psi(\widetilde{x}) \widetilde{x}<\widetilde{\rho}^{2} \quad \forall \widetilde{x} \neq 0, \text { and } \widetilde{x} \in\left(-\bar{x}, x^{r}\right)=: X_{+}
$$

where $x^{r}$ is the unique point which makes $\bar{w}>w$ for all $x>x^{r}$; and

$$
\text { for } \mu<0 \quad: \quad 0<(-\psi(\widetilde{x})) \widetilde{x}<\widetilde{\rho x}^{2} \quad \forall \widetilde{x} \neq 0, \text { and } \widetilde{x} \in\left(-\bar{x}+x^{\ell}, \infty\right)=: X_{-}
$$

where $x^{\ell}$ is the unique point which satisfies the condition $\bar{w}>w$ for all $0<x<x^{\ell}$. In general, $\rho \geq \widetilde{\rho} \geq|\mu|$ for both cases $\mu>0$ and $\mu<0$. 
Definition 5. Inspired by the discussion in [66] (p. 361), we say that the system $\widetilde{\Sigma}_{i}$, with $\psi$ satisfying (3.34) (or (3.35)), is absolutely stable if $\widetilde{x}, \dot{\widetilde{x}} \in L_{2}[0, \infty)$ for all inputs satisfying $\widetilde{u}_{i-1}, \dot{\widetilde{u}}_{i-1} \in L_{2}[0, \infty)$ and all initial conditions $\widetilde{x}(\theta) \in X_{+}\left(\right.$for $\mu>0$ ) or $\widetilde{x}(\theta) \in X_{-}$(for $\mu<0)$.

Note that when $\widetilde{x}, \dot{\tilde{x}} \in L_{2}[0, \infty)$, we have $\widetilde{x} \in L_{\infty}[0, \infty)$, and $\widetilde{x}$ is continuous with $\widetilde{x}(t) \rightarrow 0$ as $t \rightarrow 0$, see [18] (p. 186). Moreover, for the system shown in Figure 4, $\widetilde{x}, \dot{\tilde{x}} \in L_{2}[0, \infty)$ implies $\widetilde{w}, \dot{\widetilde{w}} \in L_{2}[0, \infty)$ and $\widetilde{r}, \dot{\widetilde{r}} \in L_{2}[0, \infty)$, because $\psi$ is a static nonlinearity and $2 L G(s)$ is a stable system whose impulse response is restricted to $[0, \tau]$. This observation will be used in the next section (Section 3.2.4.) when we discuss absolute stability of systems with non-zero inputs.

The system $\widetilde{\Sigma}_{1}$ shown in Figure 4 with $\widetilde{u}_{0}(t)=0$ is obtained by putting the linear time invariant system $H(s)=(s+\delta)^{-1}(2 L G(s)-1)$ in feedback with the static nonlinearity $\psi$. Note that $G(s)$ is a system whose impulse response is of finite duration. Hence $H(s)$ is stable with impulse response $h(t)$, which satisfies

$$
|h(t)| \leq \begin{cases}2 L G(0)+e^{-\delta t} & t \leq \tau \\ e^{-\delta t}\left(e^{\delta \tau} 2 L G(0)+1\right) & t>\tau\end{cases}
$$

and

$$
|\dot{h}(t)| \leq \begin{cases}\delta\left(2 L G(0)+e^{-\delta t}\right)+2 L g(t) & t \leq \tau \\ \delta e^{-\delta t}\left(e^{\delta \tau} 2 L G(0)+1\right) & t>\tau\end{cases}
$$

So, $h, \dot{h} \in L_{p}[0, \infty)$ for all $p \geq 1$. Thus $\widetilde{\Sigma}_{1}$ satisfies all the conditions required for application of the circle and Popov criterion, see e.g. $[16,17,66]$ and their references.

Applying the circle criterion, [16], for the case $\mu>0$, we see that with $\widetilde{x}(\theta) \in X_{+}$for all $\theta \in[-\tau, 0]$, the feedback system is absolutely stable if $(1-\widetilde{\rho} H(s))$ is strictly positive real, which is equivalent to having

$$
\Re\{H(j \omega)\}<1 / \widetilde{\rho} \quad \forall \omega \in \mathbb{R} .
$$

The condition (3.36) is in general less restrictive than the small gain condition

$$
\|H\|_{\infty}<1 / \widetilde{\rho}
$$

which also implies that $(1-\widetilde{\rho} H(s))$ is strictly positive real. Note that when $H(0)=\|H\|_{\infty},(3.36)$ and (3.37) are equivalent, and in this case they reduce to

$$
\frac{\alpha}{\delta}<1 / \widetilde{\rho}
$$

Clearly, this is a stronger condition than the local asymptotic stability condition, (3.1). On the other hand, if $\widetilde{\rho}=\mu$, then (3.1) and (3.38) are identical.

Now consider the case for $\mu<0$ and let us apply the Popov criterion, [16, 17, 56, 58] on the system shown in Figure 4 with $\psi$ replaced by $-\psi$ satisfying the sector condition (3.35). In this case, with the initial condition $\widetilde{x}(\theta) \in X_{-}$for $\theta \in[-\tau, 0]$, the system is absolutely stable if there exists $\widetilde{\eta} \geq 0$ such that $\widetilde{\eta}$ is not a pole of $H(s)$ and

$$
\Re\left\{\widetilde{\rho}^{-1}+(1+j \omega \widetilde{\eta}) H(j \omega)\right\}>0 \quad \forall \omega \in \mathbb{R} .
$$


Choosing $\widetilde{\eta}=\delta^{-1}+\epsilon$ for $\epsilon>0$ and letting $\epsilon \rightarrow 0$ we see that (3.39) becomes

$$
\min _{\omega \in \mathbb{R}} \Re\{G(j \omega)\}>-\frac{\delta-\widetilde{\rho}}{2 L \widetilde{\rho}}
$$

Note that, typically the left hand side of (3.40) is negative in the form

$$
\min _{\omega \in \mathbb{R}} \Re\{G(j \omega)\}=-\widetilde{K}_{G}^{-1} G(0)
$$

where $\widetilde{K}_{G}>0$ depends on the parameters of $G(s)$. Therefore, (3.40) can be satisfied only if $\delta>\widetilde{\rho}$. In conclusion, for the case $\mu<0$ and $\delta>\widetilde{\rho}$, absolute stability condition obtained from the Popov criterion is

$$
2 L G(0)<\frac{\delta-\widetilde{\rho}}{\widetilde{\rho}} \widetilde{K}_{G}
$$

where

$$
\widetilde{K}_{G}=\left|\min _{\omega \in \mathbb{R}} \frac{\Re\{G(j \omega)\}}{G(0)}\right|^{-1} .
$$

With other choices of $\widetilde{\eta}$ in (3.39) one may obtain a less conservative result, but the above particular selection gives a simple result (3.42) which allows easy comparison with local stability condition. More precisely, for the case $\widetilde{\rho}=|\mu|$, we can compare (3.42) and (3.7): the left hand sides are identical, and in general

$$
\widetilde{K}_{G} \leq k_{\max }^{o}
$$

where $k_{\max }^{o}$ is the value of $k_{\max }$ for $\eta \rightarrow 0$ in (3.6). For $G$ in the special form (2.7), for small values of $r \tau$, we have equality in (3.44) as illustrated in Figure 5.

\subsubsection{Application of the circle and Popov criterion to the $i$ th compartment for $i \geq 2$}

Now consider the second compartment $\widetilde{\Sigma}_{2}$ with input $\widetilde{u}_{1}(t)$, which is generated by $\widetilde{\Sigma}_{1}$. As discussed above, when $\widetilde{\Sigma}_{1}$ is absolutely stable, we have $\widetilde{u}_{1}, \dot{\widetilde{u}}_{1} \in L_{2}[0, \infty)$. Note that $\widetilde{\Sigma}_{2}$ is obtained by putting $H(s)$ in feedback with the static nonlinearity $\psi$; and in this case the external input is $\widetilde{u}_{1}(t)$ filtered by the stable system whose transfer function is $(s+\delta)^{-1}$. Now, if $H$ satisfies the condition (3.36) when $\mu>0$ (respectively (3.42) when $\mu<0$ ) then $\widetilde{\Sigma}_{2}$ is absolutely stable. See for example [16] (Chapter 3, in particular Section 3.12 and Exercises 3, 5 and 9), [18] (Chapter VI, section 6) and [66] (Section 6.6.2) for the extension of circle and Popov criteria to the class of infinite dimensional systems considered here with static nonlinearity and non-zero external inputs, see also [17] and references therein.

Hence, applying the circle, or Popov, criterion sequentially we see that if $\widetilde{\Sigma}_{1}, \ldots, \widetilde{\Sigma}_{i-1}$ are absolutely stable and $\widetilde{\Sigma}_{i}$ satisfies the sufficient condition (3.36) when $\mu>0$ (or (3.42) when $\mu<0$ ), then $\widetilde{\Sigma}_{i}$ is absolutely stable.

At this point we should note that stability analysis of $\widetilde{\Sigma}_{i}$ for $i \geq 2$ can also be done by applying the input-to-state-stability technique, [62], to systems with distributed time delays. In that approach, we seek a Lyapunov function to guarantee input-to-state dynamical stability (see e.g. [25]) 


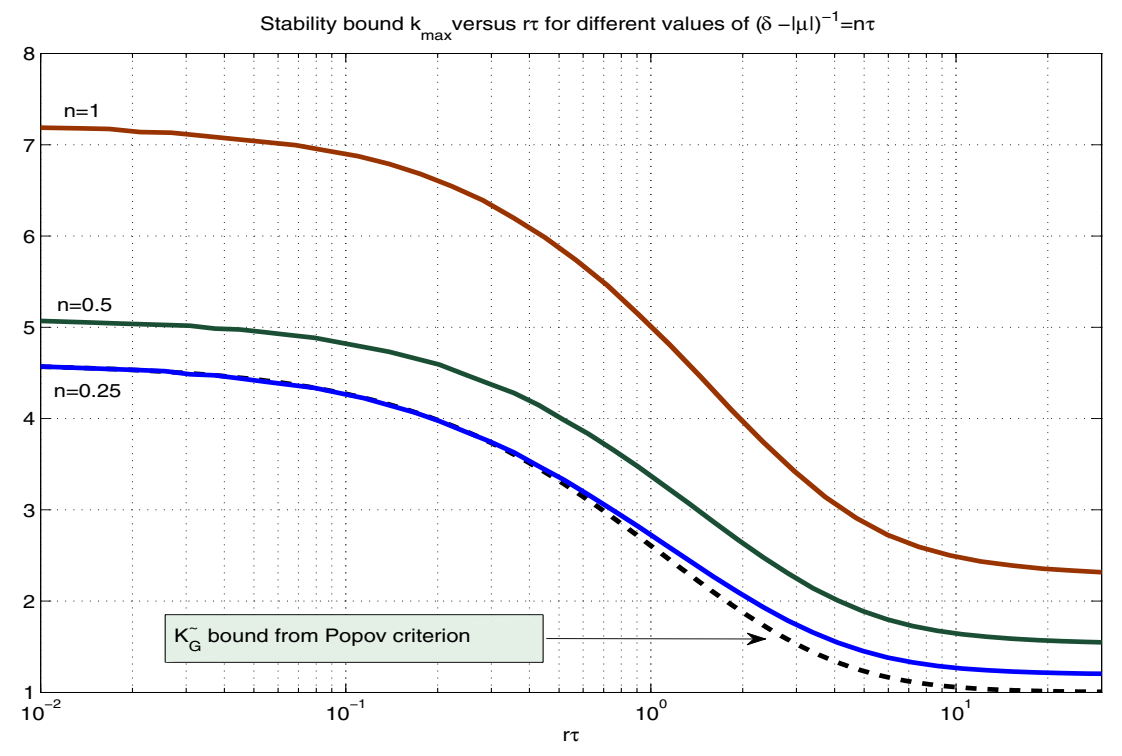

Figure 5: $k_{\max }$ versus $r \tau$ for various $(\delta-|\mu|)$; and $K_{G}$ versus $r \tau$.

of each $\Sigma_{i}$ whose inputs are known to satisfy certain boundedness condition. In fact, with the help of such a Lyapunov function, one might be able to determine a convergence rate for $\widetilde{x}_{i}(t) \rightarrow 0$. However, this requires a separate detailed study, which is beyond the scope of the present paper.

Also note that, in this section, stability conditions do not say anything about the norms $\left\|\widetilde{x}_{i}\right\|_{2}$, $\left\|\widetilde{u}_{i}\right\|_{2}$; it may be possible that these are increasing functions of $i$. These may be analyzed using the concept of "string stability" where the ratio $\frac{\left\|\widetilde{x}_{i}\right\|_{2}}{\left\|\widetilde{x}_{i-1}\right\|_{2}}$ is investigated. We leave this as an open problem for future studies. Nevertheless, we should point out that bounds on $\left\|\widetilde{x}_{2}\right\|_{2}$ and $\left\|\widetilde{u}_{2}\right\|_{2}$ can be obtained from the inequalities (3.30) and (3.32), determined in the non-linear small gain approach.

\section{Numerical Examples and Simulation Results}

In what follows we take $d$ to be the unit of time (typically, one day is the time scale of hematopoietic processes, see e.g. [24]). Hence the time delay $\tau$ is in $\mathrm{d}$ and the rates $\delta, \gamma$ and $\beta$ are in $\mathrm{d}^{-1}$. All the other parameters are normalized to have no unit. The parameter value sets do not come from a biological data; they have been chosen in such a way to illustrate instances of the different cases studied and discussed in Section 3. 


\subsection{Example 1}

As the first example we take a single compartment system with $\delta=0.2, \tau=0.66, \gamma=0.04$, $m=10, N=2, b=1, L=0.9$, and $\beta(0)=1$. We find that $\bar{x}=1.67, \mu=-0.125, \alpha=0.63$ and $k_{\max }=39.12$. In this case the sufficient condition of local stability (2.15) is not satisfied, but the necessary and sufficient condition (3.7) is satisfied.

\subsection{Example 2}

Now we consider a system with three compartments each having $L_{i}=0.9 ; \beta_{i}(0)=1 ; b_{i}=1$, and the other parameters as indicated in Table 3 . The resulting equilibrium point and computed values of $\mu, \alpha$ and other variables are shown in the second part of the same table.

\begin{tabular}{||c||c|c|c|c|c||}
\hline$i$ & $\delta$ & $\tau$ & $\gamma$ & $m$ & $N$ \\
\hline \hline 1 & 0.5 & 1.0000 & 0.04 & 10 & 2 \\
\hline 2 & 0.2 & 0.6325 & 0.04 & 10 & 2 \\
\hline 3 & 0.3 & 0.8100 & 0.50 & 9 & 3 \\
\hline
\end{tabular}

\begin{tabular}{||c|c|c|c|c|c|c|c|c||}
\hline$i$ & $\bar{x}$ & $\mu$ & $\alpha$ & $k_{\max }$ & $\rho$ & $\widetilde{\rho}$ & $h_{0}$ & $\widetilde{K}_{G}$ \\
\hline \hline 1 & 0.6876 & 0.2431 & 0.7364 & - & 0.679 & 0.679 & 0.5421 & - \\
\hline 2 & 1.9824 & -0.1206 & 0.7620 & 39.1 & 0.203 & 0.124 & 14.5287 & 1.1498 \\
\hline 3 & 0.7730 & 0.0357 & 0.2707 & - & 0.684 & 0.684 & 1.7291 & - \\
\hline
\end{tabular}

Table 3: Parameters of Example 2

For $i=1$ and $i=3$ we see that $\mu>0$ and the local stability condition $\mu_{i}<\delta_{i} / \alpha_{i}$ is satisfied. For $i=2, \mu<0$, and in this case too, the local stability condition (3.7) is satisfied (note that $2 L G(0)=\alpha+1$ ). On the other hand, the global stability condition, $\rho h_{0}<1$, is satisfied only for the first compartment. For $i=1,3$ the condition for absolute stability, (3.36), is satisfied with $x_{r}=1.45$ for the first compartment and $x_{r}=0.81$ for the third one. As for the second compartment $x_{\ell}=0.5$, but the absolute stability condition (3.42) is not satisfied. Nevertheless, starting from initial conditions $\left[x_{1}(\theta), x_{2}(\theta), x_{3}(\theta)\right]^{T}=[1.0,1.3,1.0]$ for all $\theta \in[-1,0]$, the simulations show convergence to the positive equilibrium, Figure 6.

\subsection{Example 3}

In this example we have two compartments with positive equilibrium; the parameters are $L_{1}=$ $0.985, L_{2}=0.9 ; b_{1}=b_{2}=1 ; \beta_{1}(0)=7, \beta_{2}(0)=1$ and as shown in Table 4 .

In this example we have $\mu<0$ with $|\mu|<\delta$. The necessary and sufficient condition for local stability, (3.7), is not satisfied for the first compartment and it is satisfied for the second one. Absolute stability and global stability conditions are not satisfied for both compartments. 


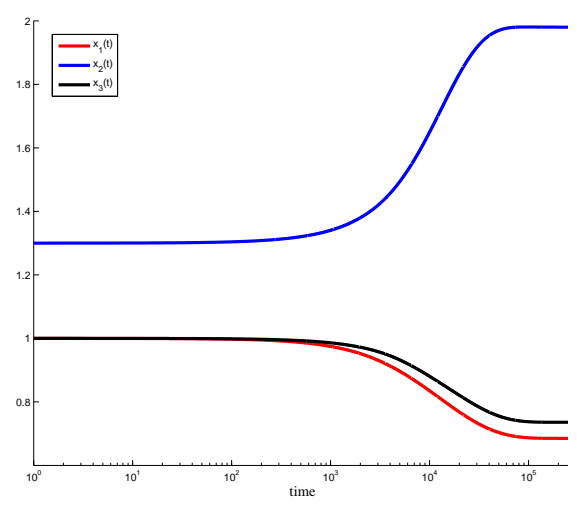

Figure 6: Simulation results for Example 2.

\begin{tabular}{||c||c|c|c|c|c||}
\hline$i$ & $\delta$ & $\tau$ & $\gamma$ & $m$ & $N$ \\
\hline \hline 1 & 2.1 & 2.81165 & 0.095 & 1 & 3 \\
\hline 2 & 0.2 & 0.6325 & 0.04 & 10 & 2 \\
\hline
\end{tabular}

\begin{tabular}{||c||c|c|c|c|c|c|c|c||}
\hline$i$ & $\bar{x}$ & $\mu$ & $\alpha$ & $k_{\max }$ & $\rho$ & $\widetilde{\rho}$ & $h_{0}$ & $\widetilde{K}_{G}$ \\
\hline \hline 1 & 1.0363 & -1.922 & 0.634 & 6.194 & 3.3128 & 2.2429 & 0.3309 & 1.66 \\
\hline 2 & 1.9664 & -0.121 & 0.762 & 39.3456 & 0.2055 & 0.1239 & 14.5287 & 1.15 \\
\hline
\end{tabular}

Table 4: Parameters of Example 3

As shown in the simulation, Figure 7, $x_{1}(t)$ does not converge to the equilibrium. Since $u_{1}(t)$ (which is the input for $\Sigma_{2}$ ) does not converge, $x_{2}(t)$ does not converge either (with small oscillations around the equilibrium).

\subsection{Example 4}

In this example we take two compartments and illustrate that the assumption $\alpha_{i}>0$ is not necessary for $i \geq 2$, in order to have a positive equilibrium. The first compartment has a positive equilibrium. The second compartment does not satisfy Assumption 2, i.e. if it was the first compartment, then the origin would be the equilibrium, but since the first one has a positive equilibrium which is globally stable, then the input to the second compartment is non-zero hence $\bar{x}_{2} \neq 0$. The parameters of this example are $L_{i}=0.9, b_{i}=1, \beta_{i}(0)=1$ for $i=1,2$ and the rest are given in Table 5. Note that the global stability condition, $\rho h_{0}<1$, holds for both compartments. Time domain response shown in Figure 8 illustrates the convergence to the equilibrium. 


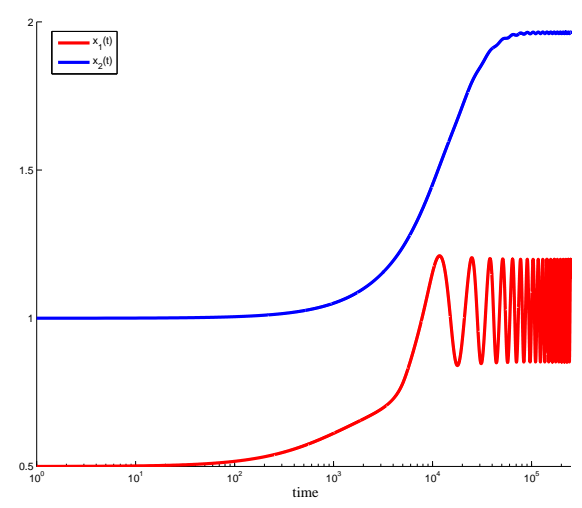

Figure 7: Simulation results for Example 3.

\begin{tabular}{||c||c|c|c|c|c||}
\hline$i$ & $\delta$ & $\tau$ & $\gamma$ & $m$ & $N$ \\
\hline \hline 1 & 0.5 & 1 & 0.04 & 10 & 2 \\
\hline 2 & 0.9 & 1.5 & 0.7 & 8 & 2 \\
\hline
\end{tabular}

\begin{tabular}{||c||c|c|c|c|c|c|c||}
\hline$i$ & $\bar{x}$ & $\mu$ & $\alpha$ & $x_{r}$ & $\rho$ & $\widetilde{\rho}$ & $h_{0}$ \\
\hline \hline 1 & 0.688 & 0.243 & 0.74 & 1.45 & 0.68 & 0.68 & 0.54 \\
\hline 2 & 0.075 & 0.98 & -0.31 & 13.4 & 0.99 & 0.99 & 0.8 \\
\hline
\end{tabular}

Table 5: Parameters of Example 4

\subsection{Remarks on simulation results}

In the above examples we have seen that when the system satisfies the local stability condition, then the graph of $x_{i}(t)$ converges to $\bar{x}_{i}$, regardless of the satisfaction of the global (or absolute) stability conditions. We tried to find an example where this convergence is dependent on the initial condition. More precisely, we would be interested in finding examples where we can make the following observations. Let $\bar{x}>0$ be the equilibrium point of a single compartment system.

- When the system is locally stable and the initial condition is in a neighborhood $X_{0}$ of $\bar{x}$, then we have convergence of $x(t)$ to $\bar{x}$. But when this system does not satisfy the absolute stability condition, if the initial condition is outside $X_{0}$, then $x(t)$ does not converge.

- When the system satisfies absolute stability condition, but not the global stability condition, if the initial condition is inside of a set $X_{1}$, which includes $X_{+}$(or $X_{-}$), then $x(t)$ converges to $\bar{x}$, but when the initial condition is outside $X_{1}$, then $x(t)$ does not converge.

Finding such examples and the corresponding $X_{0}$ and $X_{1}$ seems to be a difficult task. Many tries we have made with different numerical values turned out to be futile. 


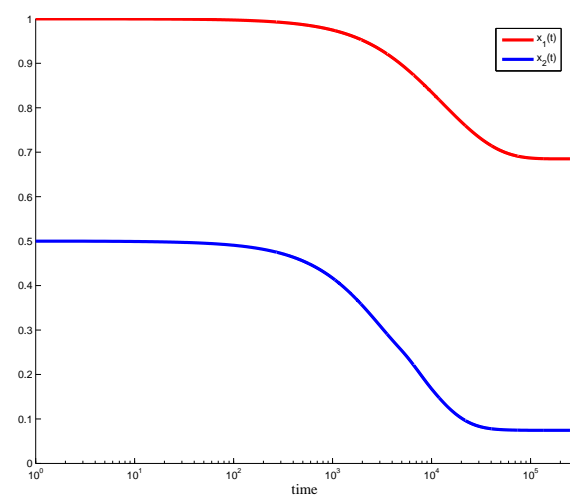

Figure 8: Simulation results for Example 4.

The above discussion leads to the following conjecture. Let $\bar{x}>0$ be the equilibrium point of a single compartment system. If the system is locally stable and all solutions remain bounded, then it is globally stable too.

Recently, it has been proven that (here we give the single compartment version of the result from [6]), when the origin is the only equilibrium, it is locally asymptotically stable if and only if

$$
(2 L G(0)-1) \beta(0)<\delta \quad \text { i.e. } \quad \beta(0)<\delta / \alpha .
$$

Moreover, the origin is globally asymptotically stable if

$$
(2 G(0)-1) \beta(0)<\delta .
$$

Note that in a single compartment model we have $K=0$ (none of the divided cells go to the next compartment) and $L=1$, hence, local asymptotic stability condition is the same as the sufficient condition for global stability. For the multiple compartment case of the lumped delay version of this system, see [57], where it has been shown that

$$
\beta_{1}(0)<\frac{\delta_{1}}{\alpha_{1}}
$$

is a sufficient condition for global asymptotic stability. The conjecture stated above deals with the extension of these results to the case $\bar{x}>0$.

\section{Conclusions}

In this paper, stability conditions are obtained for a nonlinear system with distributed delays, representing a mathematical model of cell dynamics in leukemia, that has been the subject of many earlier works [1]-[9] and [15, 22, 30, 34, 39, 49, 57, 68]. Local asymptotic stability conditions are 
obtained from the Nyquist stability criterion. Global exponential stability conditions are derived from the nonlinear small-gain type of arguments, and absolute stability conditions are obtained via the circle and Popov criteria.

All the stability conditions are expressed in terms of the parameters of the linear part, namely $\alpha, \delta, \tau, h_{0}, k_{\max }, \widetilde{K}_{G}$, and the gain of the nonlinear block $x \beta(x)$, i.e., $\mu, \rho, \widetilde{\rho}$. By adjusting some of these parameters, equilibrium and stability conditions can be changed. That can be done by taking some therapeutic actions (e.g. death rates can be adjusted by medication). Of course, periodic medications will make the system parameters vary in time; analysis of such a time varying nonlinear system with distributed delays requires a separate study. Nevertheless, for certain forms of time-varying nonlinearity, some of the analysis techniques used here remain valid, see e.g. [10]. By some other types of external intervention one may be able to reduce the leukemic stem cell (LSC) population suddenly; this corresponds to changing the initial conditions in the mathematical model. That may help bring the system within the absolute stability region near the equilibrium. This is useful when the system is absolutely stable, yet it does not satisfy the global stability condition.

Although leukemia is the best understood cancer, as far as dynamical modeling is concerned, there are still practical difficulties in determining the parameters of the mathematical model considered here. Nevertheless, stability analysis performed here can explain certain medical data. For example, it can be concluded that decreased $\gamma_{i}$ leads to instability; more precisely, decreased $\gamma_{i}$ leads to increased $G_{i}(0)$ and that may lead to violation of the inequalities (3.1), or (3.38), or (3.42). A similar observation has been made in [23] by analyzing biological data published in earlier literature on chronic myelogenous leukemia.

Other lines of future work include consideration of different types of (possibly higher order) cell division rate $f_{i}$, and experimental justification of the model using real data (e.g. from a tumor bank). We do think, and are actively and practically supported in this by medical collaborators (see Acknowledgments and references to J.P. Marie), that an interactive approach of the problem consisting in proposing, according to a physiopathologically based rationale, dynamical experiments in cell cultures, with or without added drugs, to investigate diseased cell dynamics, will result in medical advances towards understanding the disease and possibly improving its treatment. Conversely, of course, such (presently ongoing) experiments will help us identify model parameters, possibly also modify the model itself, in a more realistic way.

\section{Acknowledgements}

We thank Professor V. Rasvan for pointing out important early references on the subject, and Professor J. P. Marie for fruitful discussions on justifications of the mathematical model considered. We also thank the reviewers for suggestions which improved the paper. 


\section{Appendix: Basic Definitions from System Theory}

For the readers who are not familiar with certain basic terms from system theory, we provide a brief background material in this Appendix. Detailed treatment of these topics can be found in, e.g., $[21,50,51,66]$.

Consider the following first order differential equation with $x(t) \in \mathbb{R}$ and $u(t) \in \mathbb{R}$

$$
\dot{x}(t):=\frac{d}{d t} x(t)=A x(t)+B u(t)
$$

where $A \in \mathbb{R}$ and $B \in \mathbb{R}$ are constant coefficients. Suppose that the initial condition is zero, i.e., $x(0)=0$, and $u(t)$ satisfies $u(t)=0$ for $t<0$. In this case, the solution $x(t)$ is

$$
x(t)=\int_{0}^{t} e^{A \tau} B u(t-\tau) d \tau, \quad t \geq 0 .
$$

Therefore, $x(t)$ for $t \geq 0$ can be seen as the output due to the given input $u(t)$ for $t \geq 0$. We call such an input-output relation a system. In terms of the integration and multiplication operators the system defined by (5.1) can be represented by the block diagram shown in Figure 9.

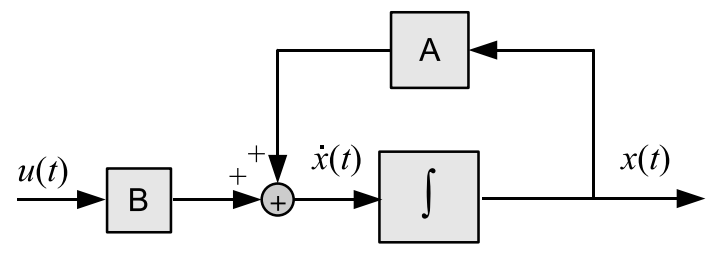

Figure 9: Block diagram representation of the system defined in (5.1).

For a given system let $x_{k}(t)$ be the output due to the input $u_{k}(t)$ for $k=1,2$; then, the system is linear if the output satisfies $x(t)=c_{1} x_{1}(t)+c_{2} x_{2}(t)$ whenever the input is $u(t)=c_{1} u_{1}(t)+c_{2} u_{2}(t)$ for arbitrary constants $c_{1}, c_{2} \in \mathbb{R}$. Let $x(t)$ be the output due to the input $u(t)$ and define $u_{o}(t)=$ $u(t-\sigma)$, for some fixed $\sigma \in \mathbb{R}$. We say that the system is time invariant if the output $x_{o}(t)$ due to input $u_{o}(t)$ satisfies $x_{o}(t)=x(t-\sigma)$ for any arbitrary $\sigma \in \mathbb{R}$. In this sense, linear differential equations with constant coefficients can be seen as linear time invariant systems.

Note that when the Dirac delta function (also called the unit impulse) $\delta(t)$ is applied as the input (i.e., $u(t)=\delta(t)$ ), in (5.1) then the corresponding output in (5.2) is $x(t)=e^{A t} B, t \geq 0$. In this sense, $h(t):=e^{A t} B, t \geq 0$, is called the impulse response of the linear time invariant system represented by (5.1). Generalizing this definition to all linear time invariant systems, we can write

$$
x(t)=\int_{0}^{t} h(\tau) u(t-\tau) d \tau, \quad t \geq 0
$$

(whenever initial conditions are zero and $u(t)=0$ for $t<0$ ) where $h(\cdot)$ is the impulse response. The Laplace transform of $h$ is defined as $H(s)=\int_{0}^{\infty} h(t) e^{-s t} d t, s \in \mathbb{C}$, is called the transfer 
function of the system. For example, when $h(t)=e^{A t} B, t \geq 0$, we have $H(s)=(s+A)^{-1} B$. Note that, in this example, the inverse is undefined at $s=-A$ : this point in the complex plane is called a pole of $H(s)$. In general, when $H(s)=(s I+A)^{-1} B$ (i.e. $A$ is a matrix) the poles are the eigenvalues of $(-A)$. In terms of the Laplace transform of (5.1) we have $(s+A) X(s)=B U(s)$. We define $(s+A)=0$ as the characteristic equation, its roots give the poles of the system. We say that $H$ is proper if $\lim _{|s| \rightarrow \infty}|H(s)|<\infty$ and it is strictly proper if $\lim _{|s| \rightarrow \infty}|H(s)|=0$. If $H(s)$ is a bounded analytic function in $\mathbb{C}_{+}$, then we write $H \in \mathcal{H}_{\infty}$ and in this case we define $\|H\|_{\infty}=\operatorname{ess} \sup _{\omega}|H(j \omega)|$ where $j:=\sqrt{-1}$. When $H(s)$ is a rational function $H \in \mathcal{H}_{\infty}$ if and only if all its poles are in $\mathbb{C}_{-}$. All these definitions extend to a class of irrational transfer functions, including systems with distributed delays as considered in Section 3.1.

There are many different definitions for the stability of a system. For the nonlinear system dealt in Section 3.2 please see Definition 2 and Definition 5. For the local stability analysis (analysis of the linearized system around the equilibrium) considered in Section 3.1, we say that the system is stable if its transfer function $H(s)$ is in $\mathcal{H}_{\infty}$.

\section{References}

[1] H. T. Alaoui, R. Yafia. Stability and Hopf bifurcation in an approachable haematopoietic stem cells model. Mathematical Biosciences, 206 (2007), 176-184.

[2] M. Adimy, F. Crauste. Modeling and asymptotic stability of a growth factor-dependent stem cell dynamics model with distributed delay. Discrete and Continuous Dynamical Systems Series B, 8 (2007), No. 1, 19-38.

[3] M. Adimy, F. Crauste. Mathematical model of hematopoiesis dynamics with growth factordependent apoptosis and proliferation regulations. Mathematical and Computer Modelling, 49 (2009), No. 11-12, 2128-2137.

[4] M. Adimy, F. Crauste, A. El Abdllaoui. Asymptotic behavior of a discrete maturity structured system of hematopoietic stem cell dynamics with several delays. Mathematical Modelling of Natural Phenomena, 1, (2006), No. 2, 1-19.

[5] M. Adimy, F. Crauste, A. El Abdllaoui. Discrete maturity-structured model of cell differentiation with applications to acute myelogenous leukemia. J. Biological Systems, 16 (2008), No. 3, 395-424.

[6] M. Adimy, F. Crauste, A. El Abdllaoui. Boundedness and Lyapunov function for a nonlinear system of hematopoietic stem cell dynamics C. R. Acad. Sci. Paris, Ser. I, 348 (2010), No. 7-8, 373-377.

[7] M. Adimy, F. Crauste, C. Marquet. Asymptotic behavior and stability switch for a matureimmature model of cell differentiation. Nonlinear Analysis: Real World Applications, 11 (2010), No. 4, 2913-2929. 
[8] M. Adimy, F. Crauste, S. Ruan. A mathematical study of the hematopoiesis process with applications to chronic myelogenous leukemia. SIAM J. Appl. Math., 65 (2005), No. 4, 1328 1352.

[9] M. Adimy, F. Crauste, S. Ruan. Modelling hematopoiesis mediated by growth factors with applications to periodic hematological diseases. Bulletin of Mathematical Biology, 68 (2006), No. 8, 2321-2351.

[10] P-A. Bliman. Extension of Popov absolute stability criterion to nonautonomous systems with delays. INRIA Technical Report No. 3625, February 1999.

[11] P-A. Bliman. Robust absolute stability of delay systems. Nonlinear Control in the Year 2000, A. Isidori, F. Lamnabhi-Lagarrigue, W. Respondek, eds., LNCIS vol. 258, Springer Verlag, 2000, 207-237.

[12] B. M. Boman, M. S. Wicha. Cancer stem cells: a step toward the cure. J. Clinical Oncology, 26 (2008), No. 17, 2795-2799.

[13] D. Bonnet, J. E. Dick. Human acute myeloid leukemia is organized as a hierarchy that originates from a primitive hematopoietic cell. Nature Medicine, 3 (1997), No. 7, 730-737.

[14] N. G. Čebotarev, N. N. Meiman. The Routh-Hurwitz problem for polynomials and entire functions. (in Russian) Trudy Mat. Inst. Steklov., 26 (1949), 3-331.

[15] C. Colijn, M. C. Mackey. A mathematical model of hematopoiesis: I. Periodic chronic myelogenous leukemia. J. Theoretical Biology, 237 (2005), No. 2, 117-132.

[16] C. Corduneanu. Integral equations and stability of feedback systems. Academic Press, New York, 1973.

[17] R. F. Curtain, H. Logemann, O. Staffans. Stability results of Popov-type for infinitedimensional systems with applications to integral control. Proc. London Math. Soc., 86 (2003), No. 3, 779-816.

[18] C. A. Desoer, M. Vidyasagar. Feedback systems: input-output properties. SIAM Classics in Applied Mathematics, 55, SIAM, 2009.

[19] D. Dingli, F. Michor. Successful therapy must eradicate cancer stem cells. Stem Cells, 24 (2006), No. 12, 2603-2610.

[20] D. Dingli, J. M. Pacheco. Modeling the architecture and dynamics of hematopoiesis. Wiley Interdisciplinary Reviews: Systems Biology and Medicine, 2 (2010), No. 2, 235-244.

[21] R. C. Dorf, R. H. Bishop. Modern Control Systems (12th Edition). Pearson Educatin Inc., New Jersey, 2011. 
[22] J. Dyson, R. Villella-Bressan, G. F. Webb. A singular transport equation modelling a proliferating maturity structured cell population. Canadian Applied Mathematics Quarterly, 4 (1996), No. 1, 65-95.

[23] P. Fortin, M. C. Mackey. Periodic chronic myelogenous leukemia: spectral analysis of blood counts and aetilogical implications. British Journal of Haematology, 104 (1999), No. 2, 336345.

[24] C. Foley, M.C. Mackey. Dynamic hematological disease: a review. J. Mathematical Biology, 58 (2009), No. 1-2, 285-322.

[25] L. Grüne. Input-to-state dynamical stability and its Lyapunov function characterization. IEEE Trans. on Automatic Control, 47 (2002), No. 9, 1499-1504.

[26] K. Gu. An improved stability criterion for systems with distributed delays. Int. J. Robust and Nonlinear Control, 13 (2003), No. 9, 819-831.

[27] P. B. Gupta, C. L. Chaffer, R.A. Weinberg. Cancer stem cells: mirage or reality? Nature Medicine, 15 (2009), No. 9, 1010-1012.

[28] T. Haferlach. Molecular genetic pathways as therapeutic targets in acute myeloid leukemia. Hematology, American Society of Hematology Educational Program, (2008), No. 1, 400411.

[29] A. Halanay. Differential Equations, Stability, Oscillations, Time Lags. (in Romanian), Editura Academiei R.P.R., Bucharest, 1963, English version by Academic Press, 1966.

[30] C. Haurie, D. C. Dale, M. C. Mackey. Cyclical neutropenia and other periodic hematological diseases: A review of mechanisms and mathematical models. Blood, 92 (1998), No. 8, 2629_ 2640.

[31] K. J. Hope, L. Jin, J. E. Dick. Human acute myeloid leukemia stem cells. Archives of Medical Research, vol.34 (2003), No. 6, 507-514.

[32] B. J. P. Huntly, D. G. Gilliland. Leukemia stem cells and the evolution of cancer-stem-cell research. Nature Reviews: Cancer, 5 (April 2005), 311-321.

[33] M. E. King, J. Rowe. Recent developments in acute myelogenous leukemia therapy. The Oncologist, 12 (2007), Suppl. 2, 14-21.

[34] L. Kold-Andersen, M. C. Mackey. Resonance in periodic chemotherapy: A case study of acute myelogenous leukemia. J. Theoretical Biology, 209 (2001), No. 1, 113-130.

[35] X. Lai, S. Nikolov, O. Wolkenhauer, J. Vera. A multi-level model accounting for the effects of JAK2-STAT5 signal modulation in erythropoiesis. Computational Biology and Chemistry, 33 (2009), No. 4, 312-324. 
[36] Z. Ling, Z. Lin. Traveling wavefront in a hematopoiesis model with time delay. Applied Mathematics Letters, 23 (2010), No. 4, 426-431.

[37] M. C. Mackey, L. Glass. Oscillation and chaos in physiological control systems. Science, 197 (1977), No. 4300, 287-289.

[38] M. C. Mackey. Unified hypothesis for the origin of aplastic anaemia and periodic hematopoiesis. Blood, 51 (1978), No. 5, 941-956.

[39] M. C. Mackey, C. Ou, L. Pujo-Menjouet, J. Wu. Periodic oscillations of blood cell populations in chronic myelogenous leukemia. SIAM J. Math. Anal., 38 (2006), No. 1, 166-187.

[40] J. P. Marie. Private communication. Hôpital St. Antoine, Paris, France, July 2010.

[41] J. A. Martinez-Climent, L. Fontan, R. D. Gascoyne, R. Siebert, F. Prosper. Lymphoma stem cells: enough evidence to support their existence? Haematologica, 95 (2010), No. 2, 293 302 .

[42] A. G. McKendrick. Applications of mathematics to medical problems. Proc. Edinburgh Math. Soc., 44 (1925), 98-130, DOI: 10.1017/S0013091500034428.

[43] W. Michiels, S. Mondie, D. Roose, M. Dambrine. The effect of approximating distributed delay control laws on stability. Advances in time-delay systems, S-I. Niculescu, K. Gu, Eds., Springer-Verlag, LNCSE 38, 2004, 207-222.

[44] F. Michor, T. P. Hughes, Y. Iwasa, S. Branford, N. P. Shah, C. L. Sawyers, M.. Nowak. Dynamics of chronic myeloid leukaemia. Nature, 435 (30 June 2005), 1267-1270.

[45] C-I. Morarescu, S-I. Niculescu, W. Michiels. Asymptotic stability of some distributed delay systems: an algebraic approach. International Journal of Tomography \& Statistics, 7 (2007), No. F07, 128-133.

[46] U. Münz, J. M. Rieber, F. Allgöwer. Robust stabilization and $H_{\infty}$ control of uncertain distributed delay systems. Topics in Time Delay Systems, J. J. Loiseau et al. Eds., LNCIS 388 (2009), 221-231.

[47] S. L. Noble, E. Sherer, R. E. Hannemann, D. Ramkrishna, T. Vik, A. E. Rundell. Using adaptive model predictive control to customize maintenance therapy chemotherapeutic dosing for childhood acute lymphoblastic leukemia. Journal of Theoretical Biology, 264 (2010), No. 3, 990-1002.

[48] S-I. Niculescu, V. Ionescu, D. Ivanescu, L. Dugard, J-M. Dion. On generalized Popov theory for delay systems. Kybernetica, 36 (2000), No. 1, 2-20.

[49] S-I. Niculescu, P. S. Kim, K. Gu, P. P. Lee, D. Levy. Stability crossing boundaries of delay systems modeling immune dynamics in leukemia. Discrete and Continuous Dynamical Systems Series B, 13, (2010), No. 1, 129-156. 
[50] A. V. Oppenheim, A. S. Willsky, H. Nawab. Signals \& Systems 2nd ed., Prentice Hall, New Jersey, 1997.

[51] H. Özbay. Introduction to feedback control theory. CRC Press LLC, Boca Raton FL, 2000.

[52] H. Özbay, C. Bonnet, J. Clairambault. Stability analysis of systems with distributed delays and application to hematopoietic cell maturation dynamics. Proc. of the 47th IEEE Conference on Decision and Control, Cancun, Mexico, December 2008, 2050-2055.

[53] H. Özbay, H. Benjelloun, C. Bonnet, J. Clairambault. Stability conditions for a system modeling cell dynamics in leukemia. Preprints of IFAC Workshop on Time Delay Systems, TDS2010, Prague, Czech Republic, June 2010.

[54] D. Peixoto, D. Dingli, J.M. Pacheco. Modelling hematopoiesis in health and disease. Mathematical and Computer Modelling, 53 (2011), No. 7-8, 1546-1557.

[55] B. Perthame. Transport equations in biology. Frontiers in Mathematics, Birkhäuser Verlag, 2007.

[56] V. M. Popov, A. Halanay. On stability for nonlinear systems with time delay. (in Russian), Automat. i Telemekhanika, 23 (1962), No. 7, 849-851.

[57] Y. Qu, J. Wei, S. Ruan. Stability and bifurcation analysis in hematopoietic stem cell dynamics with multiple delays. Physica D: Nonlinear Phenomena, 239 (2010), No. 20-22, 2011-2024.

[58] V. Răsvan. Absolute stability of time lag control systems. (in Romanian) Editura Academiei, R.S.R., Bucharest, 1975 (Russian version by Nauka, Moscow, 1983).

[59] V. Răsvan. "Lost” cases in theory of stability for linear time-delay systems. Mathematical Reports, 9 (2007), No. 1, 99-110.

[60] J. Rowe. Why is clinical progress in acute myelogenous leukemia so slow? Best Practice \& Research Clinical Haematology., vol. 21 (2008), No. 1, 1-3.

[61] N. J. Savill, W. Chadwick, S. E. Reece. Quantitative analysis of mechanisms that govern red blood cell age structure and dynamics during anaemia. PLoS Computational Biology, 5 (2009), doi:10.1371/journal.pcbi.1000416

[62] E. D. Sontag. The ISS philosophy as a unifying framework for stability-like behavior. Nonlinear Control in the Year 2000, vol.2, LNCIS 259 (2001), 443-467, DOI: 10.1007/BFb0110320.

[63] W. R. Sperr, A. W. Hauswirth, S. Florian, L. Öhler, K. Geissler, P. Valent. Human leukaemic stem cells: a novel target of therapy. European Journal of Clinical Investigation, 34, Suppl.2, (August 2004), 31-40.

[64] E. I. Verriest. Stability of systems with distributed delays. Preprints of the IFAC Conference on System, Structure and Control, Nantes, France, July 1995, 294-299. 
[65] E. I. Verriest. Linear Systems with Rational Distributed Delay: Reduction and Stability. Proc. of the 1999 European Control Conference, DA-12, Karlsruhe, Germany, September 1999.

[66] M. Vidyasagar. Nonlinear system analysis, 2nd Ed., SIAM Classics in Applied Mathematics, vol. 42, SIAM, Philadelphia 2002.

[67] M. Ważewska-Czyżewska, A. Lasota. Mathematical problems of the dynamics of a system of red blood cells. (in Polish) Matematyka Stosowana, 6 (1976), 23-40.

[68] A. Wan, J. Wei. Bifurcation analysis in an approachable haematopoietic stem cells model. J. Math. Anal. Appl., 345 (2008), No. 1, 276-285.

[69] G-M. Zou. Cancer stem cells in leukemia, recent advances. Journal of Cellular Physiology, 213 (2007), No. 2, 440-444. 